\title{
An Overview of Natural Computing Paradigms
}

\author{
Anupama Vishwas Gajbhiye ${ }^{1}$, Jaiprakash Kashinath Sirur ${ }^{2}$, \\ Triambak Ramchandra Sontakke ${ }^{3}$ \\ ${ }^{1,2,3}$ Department of Computer Science,JJT University, Jhunjhunu, Rajsthan
}

\begin{abstract}
Natural computing, also called Natural computation, is a terminology introduced to encompass three classes of methods: 1) Methods that are inspired from nature for the development of novel problemsolving techniques; 2) those that are based on the use of computers to synthesize natural=phenomenon ; and 3) those that employ natural materials such as molecules to compute. Computational paradigms studied by natural computing are abstracted from natural phenomena. The present paper presents an overview of Natural Computing Paradigms.
\end{abstract}

Keywords: Artificial Neural Networks, Evolutionary Algorithms, Swarm Intelligence, Artificial Immune Systems, DNA Computing,

\section{Introduction}

Natural Computing can be viewed as a system which represents a process happening in nature as information process occurring in nature which are used for information processing. Such processes include selfassembly, developmental processes, gene regulation networks, protein-protein interaction networks, biological transport (active transport, passive transport) networks, and gene assembly in unicellular organisms. Efforts to understand biological systems also include engineering of semi-synthetic, and understanding the universe itself from the point of view of information processing.

\section{Nature-Inspired Models Of Computation}

The most established "classical" nature-inspired models of computation are cellular automata. Neural computation and evolutionary computation. More recent computational system abstracted from natural processes includes swarm intelligence, artificial immune systems, membrane computing, and amorphous computing.

\section{Cellular Automata}

A cellular automaton is a dynamical system consisting of a two-dimensional grid of cells. Space and time are discrete and each of the cells can be in a finite number of states. The cellular automaton updates the states of its cells synchronously according to the transition rules which are given by a priori. The next state of a cell is computed by a transition rule and it depends only on its current state and the states if its neighbors.

\section{Neural Computation}

Neural computation is the field of research that emerged from the comparison between computing machines and the human nervous system. This field aims both to understand how the brain of living organisms work (brain theory or computational neuroscience), and to design efficient algorithms based on the principles of how the human brain processes information.

\section{Evolutionary Computation}

Evolutionary computation is a computational paradigm inspired by Darwinian evolution. It is a computational system based on the notion of simulated evolution. It comprises consent - or variable-size population of individuals, a fitness criterion based on random and natural selection, and includes genetically inspired operations that produce the next generation from the current one. The initial population is typically generated randomly or heuristically, and typical operators are mutation and recombination. At each step, the individuals are evaluated according to the given fitness function (survival of the fittest.) the next generation is obtained from selected individuals (parents) by using genetically inspired operators. The choice of parents can be guided by a selection operator which reflects the biological principle of mate selection. This process of simulated evolution eventually converges towards a nearly optimal population of individuals, from the point of view of the fitness function. 


\section{Swarm Intelligence}

Swarm intelligence, sometimes referred to as collective intelligence, is defined as the problem solving behavior that emerges from the interaction of individual agents (e.g., bacteria, ants, termites, bees, spiders, fish, birds) which communicate with other agents by acting on their local environments.

Particle swarm optimization applies this idea to the problem of finding an optimal solution to a given problem by a search through a multidimensional solution space. The initial set-up is a swarm of particles, each representing a possible solution to the problem. Each particle has its own velocity which depends on its previous velocity (the inertia component), the tendency towards the past personal best position (the nostalgia component), and its tendency toward a global neighborhood optimum or local neighborhood optimum (the social component).Particles thus move through a multidimensional space and eventually converge towards a point between the global best and their personal best. Particle swarm optimization algorithms have been applied to various optimization problems, and to unsupervised learning, game learning, and scheduling applications.

In the same area, ant algorithms model the foraging behavior of ant colonies based on stigmergy. To find the best path between the nest and a source of food, ants rely on indirect communication by laying a pheromone trail on the way back to the nest if they found food, respectively following the higher concentration of pheromones if they are looking for food. Ant algorithms have been successfully applied to a variety of combinatorial optimization problems over discrete search spaces.

\section{Artificial Immune Systems}

Artificial Immune systems (also called as immunological computation or immune-computing ) are computational systems inspired by the natural immune systems of biological organisms. An artificial immune system is used in the design of fault tolerant systems.

\section{Membrane Computing}

Membrane computing investigates computing models abstracted from the compartmentalized structure of living cells which are affected by phenomena of membranes such are absorption, percolation and transmission. A generic membrane system also called a P-system consists of cell-like compartments (regions) delimited by membranes, that are placed in a nested hierarchical structure. Each membrane is an enveloped region contains object, transformation rules which modify these object, as well as transfer rules, which specify whether the object will be transferred outside or stay inside the region. Regions communicate with each other via the discrete transfer of objects.

\section{Amorphous Computing}

In biological organisms, morpho-genesis (the development of well- defined shapes and shape grammar is defined using functional structures).It is achieved by the interactions and production rules used between cells which guide by the genetic program encoded in the organism's DNA.

\section{Systems Biology}

Computational systems biology (or simply systems biology ) is an integrative and qualitative approach that investigates the complex communications and interactions taking place in biological systems. Thus in systems biology, the focus of the study are the interaction networks themselves and the properties of biological systems that arise due to these networks, rather than the individual components of functional processes in an organism. This paradigm is focused on organic components which has four different interdependent interaction networks : gene-regulatory networks, biochemical networks, transport networks and carbohydrate networks.

\section{Conclusion}

Natural Computing is a branch of Theoretical Computer Science which is useful for creating Mathematical and Computational models which are based on the philosophy of Nature. In the present paper we have presented an overview of Natural computing paradigms and their applicability to solve real world applications.

\section{Acknowledgements}

The authors of the paper are thankful to all the staff members of Department of Electronics and Computer Science, R.T.M. Nagpur University, Nagpur for providing Library and Laboratory for conducting the project work. 


\section{References}

[1]. Abelson H. Allen, D., Coore, D., Hanson, C., Homsy, G., knight Jr. T., Nagpal, R., Rauch, E., Sussman., Weiss,. R. Amorphous computing of the ACM 43, 5 (May 2000), 74-82

[2]. Adleman, L., Molecular computation of solutions to combinatorial problems. Science 266 (1994), 1021-1024

[3]. Angeleska, A.,Jonoska, N., Saito, M.: Landweber., L., (2007). "RNA-guided DNA assembly". Journal of Theoretical Biology 248: 706-720, doi : 10.1016/j.jtbi.2007.06.007.

[4]. Arbib, M., The Handbook of Brain Theory and Networks. MIT press, 2003.

[5]. Black, T., Fogel, D., Michalewicz., editors. Handbook of Evolutionary Computation. IOP Publishing, U.K., 1997

[6]. Brooks., R. Artificial life : from robot dreams to reality. Nature 406 (2000), 945-947

[7]. Cardelli, L. Abstract machines of systems biology Bulletin of the EATCS 93 (2007), 176-204

[8]. Cardelli, L. Brane calculi: Interactions of biological membranes. In LNCE 3082, pages 257-280. Springer, 2005.

[9]. Dasgpuat D., editor. Artificial immune systems and Their Applications. Springer, 1998

[10]. De Castro, L.N.,Fundamentals of Natural Computing Basic Concepts, Algorithum, and Applications, CRC Press, 2006

[11]. De Castro, L., Timmis., J. Artificial immune Systems: A New Computational Intelligence Approach. Springer., 2002

[12]. Engelbrecht, A. Fundamentals of Computational Swarm Intelligence. Wiley and Sons, 2005.

[13]. Fredkin, F. Digital mechanice : An informational process based on reversible unuiversal CA, Physica D 45 (1990) 254-270

[14]. Fujibayashi, K., Hariadi, R. Park, S-H., Winfree, E., Murata, S. Toward reliable algorithmic self-assembly of DNA tiles : A fixedwidth cellular automaton pattern. Nano Letters 8 (7) (2007) 1791-1797.

[15]. G. Rozenberg. T. Back J. Kok, Editors, Handbook of Natural Computing, Sringer Verlag. 2012

[16]. Hirvensalo. M. Quantum Computing, $2^{\text {nd }}$ Ed. Springer, 2004

[17]. Istrail. S., De-Leon, B-T., Davidson, E The regulatory genome and the computer. Developmental Biology 310 (2007). 187-195

[18]. Kari, L., DNA computing - the arrival of biological mathematies. The Mathematical Intelligencer 19, 2 (1997) 9-22

[19]. Kohn, K. Molecular interaction map of the mammalian cell control and DNA repair systems. Molecular Biology of the Cell 10 (8) (1999) 2703-2734.

[20]. Koza, J. Genetic Programming: on the Programming of Computers by Means of Natural Selection. MIT Press, 1992 